\title{
EL MODELO TECNOLÓGICO DE INTERVENCIÓN PSICOPEDAGÓGICA
}

\author{
THE TECHNOLOGICAL MODEL \\ OF PSYCHO-PEDAGOGICAL INTERVENTIONS
}

\author{
Antonio Pantoja \\ Universidad de Jaén
}

\section{RESUMEN}

En este artículo se lleva a cabo una aproximación conceptual al denominado Modelo Tecnológico (MT) de intervención psicopedagógica en orientación.

En una primera parte se enmarca el modelo en el escenario de la Sociedad de la Información y se revisan algunas notas definitorias del mismo aparecidas a lo largo de los últimos años en la literatura especializada en orientación, que siempre han tenido un carácter meramente teórico.

Seguidamente, se analiza el MT desde distintos ángulos y puntos de vista, se establecen sus características principales y se desarrolla el marco metodológico que le es propio para su correcta implementación en la acción orientadora. Por último, se resumen las ventajas e inconvenientes que presenta.

Palabras clave: Modelo tecnológico, nuevas tecnologías, orientación, intervención, Sociedad de la Información.

\begin{abstract}
In this article, we can find a conceptual approach to the so-called Technological Model (TM) of psycho-pedagogical intervention in the field of Guidance.

In the first part, the model is set in the framework of the Information Society and a revision of some defining notes of this model appeared through the last years in the specialised publications on Teaching Guidance, is carried out which have always had a merely theoretical character.

Then, the TM is analysed from different angles and points of view, its main features are established and the methodological framework that is necessary for its adequate implementation within the guiding action in the teaching process is also developed. Finally, the advantages and shortcomings that this model presents are summarised.
\end{abstract}

Key words: Technological Model, New Technologies, Guidance, intervention, Information Society. 


\section{Introducción}

En la actualidad es una demanda creciente que la orientación utilice las Nuevas Tecnologías de la Información y la Comunicación (NTIC) en aras de una intervención más eficaz, de una mayor calidad y más cercana a la persona y a los retos de una sociedad cada vez más tecnologizada. Avala esta idea la inclusión de las NTIC en los distintos eventos que se celebran en todo el mundo en materia educativa y, de manera particular, sobre orientación, así como en las conferencias que sobre este tema específico vienen teniendo lugar en distintos países europeos desde hace ya varios años. La última, « $5^{\text {a }}$ Conferencia Europea sobre Tecnologías de la Información y la Comunicación en Orientación», celebrada en junio del año 2001 en Goteborg (Suecia), trató el lema de Calidad y ética en la orientación por red, un título que nos parece sugerente, por cuanto avanza sobre la idea cada día más asentada de una orientación que debe llegar a todos los usuarios y a todos los rincones del planeta a través de la red, pudiendo paliar los obstáculos que encuentran determinadas sociedades menos desarrolladas para acceder a las tecnologías (Delors y otros, 1996).

El nuevo escenario social en el que se desarrollará la orientación del futuro estará presidido plenamente por las NTIC. Los autores que se han ocupado de este tema, con la finalidad de delimitar los distintos elementos que le dan forma, no han llegado a un consenso sobre la manera de referirse al mismo. Así, han surgido nombres como Mundo Digital (Negroponte, 1995), Sociedad Digital (1996), Sociedad Red (Castells, 1997, 1998), Tercer Entorno (Echeverría Ezponda, 1999), por citar sólo algunos de ellos. Sin embargo, los términos más extendidos están siendo Sociedad del Conocimiento y, de manera más destacada, Sociedad de la Información (Telefónica, 2001). Esta diversidad no hace sino ponernos alerta de que se trata de un entorno aún por constituir, de un complejo entramado de aspectos tecnológicos y necesidades o demandas de la sociedad que crece y se estructura alrededor de ellos. Nuestras pretensiones a lo largo de este artículo serán abrir caminos, desbrozar la multitud de elementos tecnológicos que vienen adosados a este nuevo modelo de sociedad y buscar sus aplicaciones a la orientación. La formación de las personas, tanto inicial como continua, está cada día más unida a las NTIC y, por consiguiente, cercana al proceso de orientación para la vida que tanto persigue el sistema educativo. Estamos cada vez más cerca de ver cómo la orientación mediante el uso de NTIC es capaz de mejorar su calidad, así como la del trabajo del orientador, de forma que éste podrá disponer de más tiempo para el desarrollo de otras tareas como el consejo, relegadas a veces a un segundo plano.

Pero volvamos, aunque sólo sea de manera sucinta, al concepto de Sociedad de la Información (SI). En realidad, podríamos decir que se trata de una forma evolucionada de la sociedad moderna caracterizada por un desplazamiento de paradigma en las estructuras industriales y en las relaciones sociales. Al igual que la revolución industrial supuso una profunda modificación de las sociedades agrarias en el último cuarto del siglo XIX, la SI designa una forma nueva de organización de la economía y de la sociedad.

Existen una gran multiplicidad de significados que se atribuyen a la SI, de forma que no se ha llegado a ninguna definición que se pueda reconocer aceptada comúnmente. Desde nuestro punto de vista, la SI constituye una forma de desarrollo social basada en el uso habitual de las NTIC por todos los ciudadanos a nivel individual y colectivo, público y privado, para obtener, tratar y compartir información de forma instantánea desde cualquier lugar, tiempo y forma definidos previamente por sus usuarios. 
En la SI se da más importancia ya a la globalización del conocimiento que a la economía (Rodríguez Roselló, 2001). Esto implica un marco sociocultural que demanda de manera creciente nuevos perfiles profesionales basados en el uso de las NTIC. Su consecuencia directa es la distinción entre los participantes en la cohesión social -dominadores de los recursos tecnológicos- y los que empiezan a encontrar una primera vía de exclusión como consecuencia de no disponer de acceso a la información. Este déficit de competencia afecta a la población en general y a los propios profesionales para dar satisfacción a la demanda actual de tecnologías, y tiene especial relevancia en las tecnologías del empleo, al dedicar las empresas recursos a la formación de sus empleados, aunque el $50 \%$ del mercado se centra en la enseñanza de NTIC (Rodríguez Roselló, 2001).

Para hacer frente a los cambios que la SI precisa se ha constituido a nivel europeo una Dirección General para la Sociedad de la Información, dentro de las competencias de la Comisión Europea. En nuestro país existe desde hace unos años un Ministerio de Ciencia y Tecnología que está emprendiendo distintas iniciativas en todos los sectores implicados. A modo de ejemplo, destaca el Programa de Fomento de la Investigación Técnica (PROFIT), convocado en febrero de 2001, que incluía entre las áreas científico-tecnológicas el «Programa Nacional de Tecnologías de la Información y las Comunicaciones» y dentro de éstas el área sectorial de «Programa Nacional de la Sociedad de la Información», en el marco de la «Iniciativa Estratégica del gobierno para el Desarrollo de la Sociedad de la Información (INFO XXI)».

En definitiva, son muchas las acciones de envergadura y de consistencia que se están emprendiendo desde las distintas Administraciones para adecuar a las estructuras y a las personas a las demandas de la SI. Aunque, tal vez la iniciativa que más repercusión pueda tener en la orientación sea la denominada e-learning, que se inscribe en el contexto educativo. Se trata de un ingente esfuerzo económico que llevará los ordenadores en red a todas las aulas de la Europa comunitaria. La orientación deberá salir beneficiada de la llegada masiva a los centros de recursos tecnológicos en red y, al mismo tiempo, deberá prepararse para afrontar los cambios que se producirán en al alumnado por los efectos de las NTIC.

Diversos autores se han referido al reto que para la educación supone la SI (Negroponte, 1995; Terceiro, 1996; Fernández, 1998; Levy, 1999), entre las novedades destacan las representaciones virtuales, la capacidad interactiva, la simulación en tiempo real, su enfoque global o su proyección hacia las relaciones interpersonales y la participación. Este vasto panorama se abre no sólo a la educación, también a la orientación, y permite, entre otras cosas, disponer de potentes herramientas en el proceso de aprendizaje, acercar más al alumnado a su futuro fuera del sistema educativo y, quizás lo más importante, recoger procesos dinámicos que se estaban desarrollando en la sociedad.

En esta línea, la orientación en sus distintos niveles y perspectivas habrá de realizar un esfuerzo para poder desarrollarse adecuadamente en los contextos en los que tiene lugar. Sólo así podrá dar respuestas a los cambios de esta nueva sociedad sin fronteras que se está constituyendo (Cogoi, 2002). Pero para hacerlo, la acción orientadora deberá adecuarse al marco de competencias que exige la SI. Un problema que afecta de igual forma a la educación. Como afirma Echeverría Ezponda (2001), por el mero hecho de introducir las NTIC en los contextos de trabajo no tienen por qué producirse mejoras en los mismos. Para que esto ocurra deberán adecuarse las estructuras, la formación de los agentes implicados (orientadores) y diseñar un marco metodológico adecuado. Esto último es lo que se ha con- 
venido en denominar tímidamente Modelo Tecnológico de intervención psicopedagógica, pero sin ahondar en las peculiaridades que lo elevan realmente al rango de modelo. Esta será nuestra finalidad en las líneas que siguen.

\section{EI Modelo Tecnológico (MT) en la literatura sobre orientación}

Han sido muchos los autores que han referenciado el MT como tal en los últimos años (Rodríguez Espinar y otros, 1993; Álvarez Rojo, 1994; Álvarez González, 1995; Bisquerra y Álvarez González, 1996, 1998; Martínez González, 1998; Benavent y Fossati, 1998; Benavent, 1999; Lázaro y Mudarra, 2000; Sobrado y Ocampo, 2000; Pantoja, 2001; Pantoja y Campoy, 2001), mientras que otros han planteado sólo el uso de las NTIC en la orientación sin llegar a hablar de modelo (Repetto y otros, 1994; Marco, 1995; Repetto y Malik, 1998; Solé, 1998; Vélaz de Medrano, 1998; Álvarez González y Rodríguez Espinar, 2000, 2001; Marín y Rodríguez Espinar, 2001). A pesar de esta abundancia bibliográfica no se encuentra una delimitación del MT equiparable al resto de los modelos. Esta precariedad epistemológica proviene de un inseguro surgimiento a expensas de la educación, la falta de una tradición consolidada al respecto y las todavía limitadas aplicaciones específicas al campo de la orientación. En cualquier caso, ya es tiempo de que se concrete y afiance entre los orientadores el uso sistemático de las NTIC y se integren en un modelo de trabajo concreto, o lo que es igual, se dé cobertura teórico-práctica al MT.

Al dar respuesta a esta necesidad indiscutible, la orientación podrá afrontar con éxito las grandes transformaciones tecnológicas, económicas y sociales que se están operando como consecuencia de (Pantoja y Campoy, 2001):

- La creciente disponibilidad de canales de información, en la que la orientación juega el papel de desarrollar estrategias para el uso de la información (búsqueda, asimilación, organización, análisis, resolución de problemas, toma de decisiones, etc.) como competencias sociales y comunicativas.

- Una sociedad más plural, dinámica e interactiva en la que los estudiantes necesitan organizar su vida no sólo académica sino también profesional y personal.

- La necesidad de formación continuada e independiente, así como el desarrollo de habilidades de planificación de este proceso.

- La mayor validez del principio de aprender a aprender.

- La aparición continua de nuevas profesiones en el mercado laboral.

- El incremento de la complejidad y asiduidad de los procesos de elección en relación a la configuración del propio currículum.

- La alternancia trabajo-estudio.

- La necesidad de conocimiento de sí mismo y desarrollo de la autoconfianza y proyección profesional.

- La irrupción de nueva relación entre enseñanza y aprendizaje fuera del espacio y tiempo real.

Las contribuciones de diferentes ciencias han sido parte fundamental en la concepción de la tecnología educativa utilizada por la orientación, y cada vez es más frecuente el diseño y la construcción de propuestas específicas de intervención que utilizan como soporte 
básico dichas aportaciones para realizar procesos de intervención orientadora (por ejemplo, aplicaciones informáticas diseñadas para informar o para ofrecer un amplio centro de recursos, programas de televisión, vídeos específicos para la búsqueda de empleo, etc.).

Una interesante aportación con respecto a las posibilidades de las NTIC en la escuela y su repercusión en todos los ámbitos educativos nos la ofrece Marcelo (2000: 403), quien ve en las mismas una evolución social a la que tiene que ser receptiva la escuela. Defiende la innovación que suponen las NTIC en todos los ámbitos educativos, pero de forma más significativa en las relaciones de cooperación y de comunicación, citando de manera especial el soporte telemático, muy indicado en el intercambio de experiencias. Nos parece significativo que este autor defienda que la simple incorporación de las nuevas tecnologías en las escuelas no garantice la efectividad de los resultados alcanzados, por lo que es preciso integrarlas en un diseño global y una teoría que las justifique y delimite. Esta estructura epistemológica y conceptual la ofrecería en nuestro campo el MT.

La referencia más antigua al MT la encontramos en Rodríguez Espinar y otros (1993: 181-182), quienes, tras incluirlo como un modelo de orientación más, expresan lo siguiente:

"Este modelo basado en sistemas y/o programas autoaplicables no elimina la figura y las funciones del orientador. El orientador habrá de estar presente en el proceso desempeñando la función de consultor, aclarando dudas, resolviendo problemas, comentando algunas de las informaciones que se proporcionan y ayudando al sujeto en su labor de síntesis y de reflexión. Estos sistemas lo que pretenden es liberar al orientador en tareas informativas y le dejan más libre para desempeñar sus funciones de consulta y asesoramiento. Este modelo, plenamente realizado, puede contribuir en el desarrollo de las funciones de la interacción orientadora».

De igual forma, nos parecen especialmente indicadas las palabras de Benavent (1999: 60-61):

"A largo plazo, aparecerán modelos tecnológicos que sustituirán definitivamente los modelos básicos, al permitir bancos de datos de las redes informáticas y telemáticas, dar respuestas satisfactorias a las necesidades individuales más especificas y desarrollar realidades virtuales».

En el mundo de las NTIC hablar de largo plazo es excesivo. Baste con volver la mirada a la evolución de las mismas, a su rápida introducción en todos los ámbitos sociales y a la continua aparición de nuevos desarrollos y aplicaciones. En este sentido nos alerta de manera especial la fuerte apuesta de la Unión Europea por introducirlas en la educación. El plan e-learning, al que aludíamos líneas atrás, llevará de forma masiva equipos y redes telemáticas a los centros en una progresión ya imparable y en un corto espacio de tiempo. Ante esto caben algunas preguntas ¿qué actitud adoptará el orientador y el Departamento de Orientación?, ¿qué modelo de trabajo utilizará en la intervención orientadora?

Lo que está fuera de dudas es el valor que cobra la información en el MT debido a su facilidad de difusión en la SI. En este sentido, afirma Castells (1997: 94):

«El surgimiento de un nuevo paradigma tecnológico organizado en torno a nuevas tecnologías de la información y la comunicación más potentes y flexibles, hace posible que la misma información se convierta en el producto del proceso de producción». 
Pensamos, pues, que es la información -también el conocimiento- el componente esencial al que deberá dar respuesta el MT a través de nuevos procesos orientadores comandados por las NTIC aplicadas de forma especializada a la orientación.

Sin embargo, una tendencia bastante arraigada entre algunos autores que han realizado aportaciones en torno al MT, es equiparar éste al simple hecho de utilizar NTIC en la orientación. Así, Bisquerra y Álvarez González (1996: 101) han señalado:

«Entre las propuestas recientes en orientación profesional cabe citar los recursos informáticos. Existen programas informáticos, como el SIGI, Discover, Microskills, Choices, Prospect, etc., que suponen la introducción de la tecnología informática en orientación. Esto permite hablar de un nuevo modelo tecnológico en orientación que amplía el campo de la intervención».

Como veremos seguidamente, la formación de un modelo obedece a cánones de trabajo estructurado más complejos que la simple aplicación de un instrumento o herramienta concretos. No obstante, como punto de partida nos han servido para abrir el camino de la innovación.

Existen autores que se han pronunciado al respecto, exponiendo las necesidades futuras en materia de NTIC. Tal es el caso de Sancho (2000: 315) cuando afirma:

«La urgencia de diseñar entornos de aprendizaje, virtuales o presenciales, en los que todos los ciudadanos puedan encontrar una lugar en el que desarrollar y adquirir los conocimientos y las destrezas que les permitan ser mejores personas, ciudadanos y ciudadanas más responsables y comprometidos y trabajadores más cualificados».

Para favorecer esto es necesaria una definición y delimitación lo más precisa posible del MT y de los desarrollos realizados utilizando las NTIC asociadas al mismo, con el fin de contribuir a que las acciones que se emprendan en los años venideros no recorran caminos erróneos ni incurran en errores provocados por la falta de un referente concreto de acción.

\section{EI Modelo Tecnológico frente al resto de los modelos}

La estrecha relación que el MT guarda con el resto de los modelos hace que éste pueda ofrecer una amplia cobertura en casi todos los aspectos significativos de los mismos. Ahora bien, para que esto ocurra habrá de constituirse formalmente. Tras esta presunción existe una firme convicción de los servicios que en un futuro podrá aportar. En cualquier caso, el MT no desbancará a los demás, puesto que como afirman Rodríguez Espinar y otros (1993: 184), será necesaria una sincronización de diversas funciones asociadas a los diferentes modelos (relación personal, consulta, utilización de medios tecnológicos, etc.) para conferir a la orientación el carácter integral que precisa.

El orientador será el que sufrirá la transformación necesaria para poder ejercer sus funciones en tan amplio abanico de posibilidades, desempeñando roles muy diversos y utilizando instrumentos variados. Su labor será por igual en el centro (u otros contextos) y en los entornos de vida de los sujetos orientados (familias, barrios, lugares de ocio, etc.). $\mathrm{Su}$ principal armazón técnico lo formarán los programas comprensivos en unos formatos abiertos y flexibles, permitiendo dinámicas de investigación-acción. 
Bajo nuestro punto de vista, el referente de la intervención y el que dicte los pasos y naturaleza de la misma será el modelo que se toma como base de la acción, integrándose en el mismo el MT (gráfico 1).

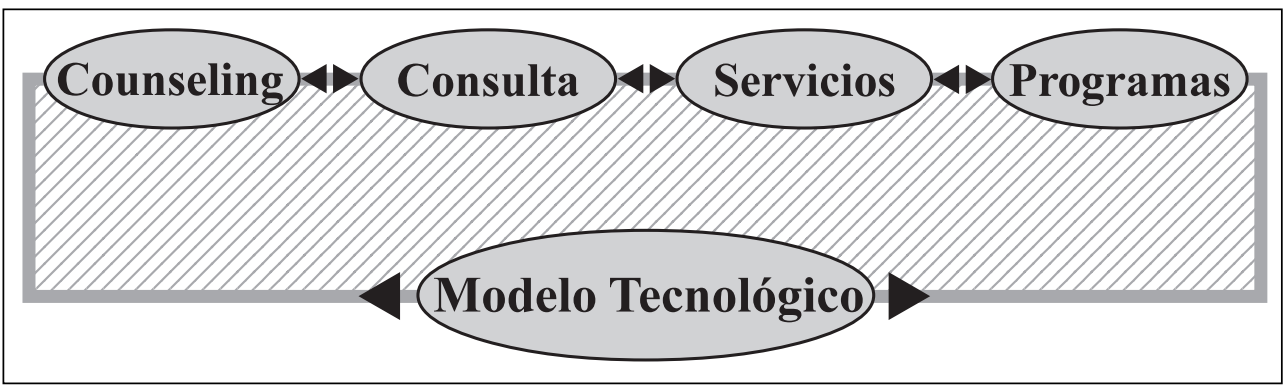

GRÁFICO 1.

Niveles de integración del MT con el resto de modelos.

A continuación, analizaremos el tipo de relaciones que se establecen entre el MT y los demás modelos:

- Counseling: La acción directa a escala individual o en grupos reducidos se puede potenciar mediante el uso de recursos tecnológicos a través de programas para tratamiento específico de determinados problemas, sean de aprendizaje, de tipo conductual, afectivo, social, etc. En ocasiones estos programas se inscriben más en una línea didáctica que orientadora. Nuestra posición es que el orientador coordine las intervenciones de tipo remedial, ya sea llevándolas a cabo él mismo o estableciendo las pautas que deberá seguir el tutor. En cualquier caso, esta acción remedial mediante NTIC puede ser implementada en el centro o en las casas, estando el estudiante sólo o en compañía de sus padres o compañeros de clase. La acción directa del orientador o del tutor, según los casos, podrá ser presencial -trabajo en el aulao a través del correo electrónico -tutoría telemática-. Al tutor corresponde en este enfoque el diseño del programa y la coordinación del mismo. La calidad de los recursos tecnológicos utilizados será la que marque la forma que adoptarán los agentes de la intervención.

- Consulta: En este modelo se pone de manifiesto la formación del orientador en NTIC. Al ocupar una posición de coordinador o colaborador, precisa de conocimientos técnicos para ayudar al profesor o al alumno, según los casos. La característica diferencial más notoria del MT respecto de la intervención consultiva es el tipo de relación que se establece entre los implicados y el hecho de que la mediación la realice el recurso tecnológico (objeto o sistema). En las relaciones de consulta existen grandes posibilidades de intervención mediante los recursos tecnológicos, al tratar éstos sobre temáticas que invitan más a la prevención y al desarrollo que a los tratamientos específicos de dificultades, como veíamos en el modelo de counseling. Una mención aparte merece la función de consulta dirigida a padres sobre aspectos relacionados con las NTIC, como pueden ser programas de televisión, videojuegos, software educativo, etc. 
- Servicios: Sus funciones, cercanas en muchos casos al modelo de counseling, hacen que su relación con el MT adopte similares características que éste. Las NTIC que más cabida presentan cuando se adopta una perspectiva de Servicios son las redes telemáticas, que facilitan un acercamiento de los mismos y una integración, en este caso virtual, en la estructura de los Departamentos de Orientación. La cobertura de los distintos problemas que surjan en el centro, el envío de documentos, la aplicación de cuestionarios u otro tipo de pruebas, la conexión con los orientadores, etc. encuentran vías de mejora a través del uso de las distintas herramientas de comunicación y transferencia de datos que incorpora Internet. Podríamos decir que con el MT el Modelo de Servicios es un poco menos externo a los centros.

- Programas: Al ser este modelo una superación del anterior, permite una adecuación más directa con el MT. Así, se encuentran en el mercado gran cantidad de programas que basan su funcionamiento en las NTIC. Al haber sido evaluados algunos de ellos, se conoce su efectividad real. Ahora bien, lo importante en la conjunción de ambos modelos es que exista una auténtica integración. Desde nuestro punto de vista, ésta sería la mejor forma de intervención posible. No basta sencillamente con entregar al alumno el programa informático, el vídeo o la dirección de Internet para que el proceso orientador transcurra por los cauces adecuados, sino que es preciso realizar un diseño claro y concreto de la intervención orientadora y de los lugares que ocupa cada recurso en la misma, así como los roles a desempeñar por los distintos agentes de intervención. Nos ocuparemos con más detalle de estos aspectos cuando en el siguiente apartado veamos las notas características del MT.

Como hemos visto, el MT ofrece características nuevas, diferentes perspectivas y posibilidades a cada uno de los modelos de orientación. La clave a despejar en el horizonte que abre en la orientación es cómo realizar su integración en las formas tradicionales de intervención. Esta será la próxima parada en nuestro camino. Pero antes, es conveniente precisar la posición del MT en relación con las cuatro grandes categorías de intervención: directa-indirecta o individual-grupal (gráfico 2).

\section{Definición y características generales}

\section{Orígenes y fundamentos del modelo}

Los inicios del MT son los propios de un sistema basado en unas tecnologías que en los primeros años no estuvieron exentas de los mismos problemas que atañen a todo nuevo sistema que quiere abrirse paso. La referencia más remota la encontramos en EE.UU. en los años sesenta cuando empieza a tomar forma una tímida introducción de los ordenadores en ámbitos del desarrollo de la carrera y la toma de decisiones (Harris-Bowlsbey, 1995). En estos tiempos todavía eran muy rudimentarios y costosos los equipos informáticos y tenían muy poca operatividad. En cualquier caso estamos hablando de experiencias aisladas.

Programas como SIGI y SIGI PLUS o el DISCOVER (American College Testing Program) fueron los primeros intentos serios de extender masivamente el uso del MT en las décadas de los setenta y ochenta. Entre los obstáculos que frenaron su expansión definitiva se 


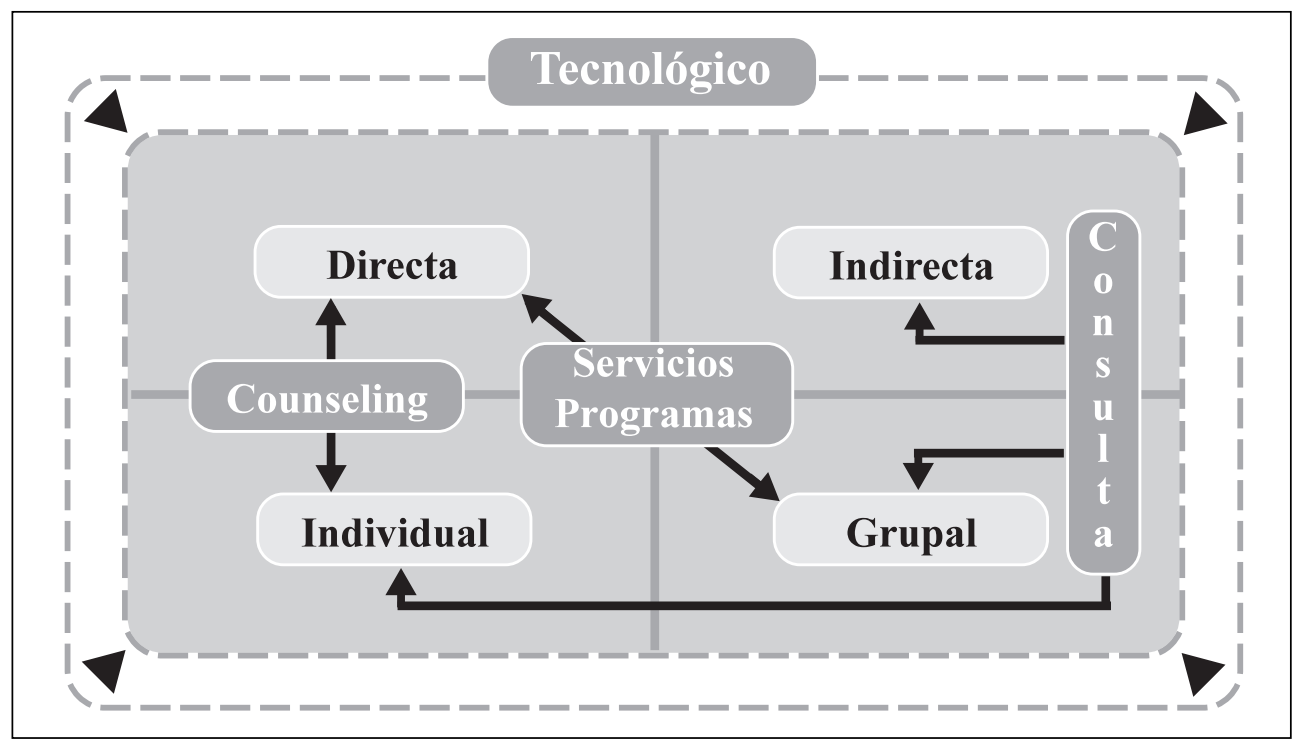

GRÁFICO 2.

Categorías definitorias de los modelos de orientación e intervención.

pueden citar: su alto coste, la dependencia del fabricante y la falta de un refrendo empírico sobre su eficacia. Estos y otros inconvenientes provocaron un período de reflexión sobre el lugar de estas herramientas en el proceso de ayuda a la persona (Marín y Rodríguez Espinar, 2001).

En la década de los ochenta la orientación norteamericana-como se ve, nuestro principal referente- presentaba una serie de rasgos identificativos comunes entre los que destacaba el aumento del uso de ordenadores y otros adelantos tecnológicos por parte de los orientadores (Daniel y Weikel, 1983).

Herr y Cramer (1992) señalaron en un estudio algunas de las características que definían, según ellos, la orientación de finales de los años ochenta y la de comienzos de los noventa, entre las que destaca la utilización de tecnología informática en los procesos de orientación. Eran estos unos años de mejoras técnicas importantes que dieron lugar al abaratamiento de los equipos y su introducción en los centros educativos. Esto favoreció la aparición de los primeros programas desarrollados de forma específica para su uso por los orientadores.

Antes de adentrarnos en una revisión histórica más exhaustiva, es preciso hacer referencia a las dos constantes que marcan los inicios y el posterior desarrollo del MT:

1. Las escasas referencias a los medios audiovisuales en la orientación.

2. El alto interés despertado por la tecnología informática en todos los estudios (Sampson y Reardon, 1991; Rayman, 1990; Harris-Bowlsbey, 1995; Marco 1995).

Estas razones nos inducen a ocuparnos de forma preferente de las tecnologías informáticas, que en su aplicación a la orientación han seguido una línea de desarrollo ascendente a 
la par que los avances técnicos, yendo desde las primitivas aplicaciones a la corrección de test y pruebas de todo tipo a los sistemas multimedia interactivos de orientación vocacional. En total se puede afirmar que han existido cinco grandes generaciones en el desarrollo de las NTIC (Sampson y Reardon, 1990; Rayman, 1990; Marco, 1995).

La primera estuvo maniatada por el escaso desarrollo de los ordenadores, de los que aprovecharon su potencia de cálculo para corregir test. Apenas si encontramos referencias en esta época en la que las tareas de orientación se basan en la comparación de resultados de cuestionarios convencionales con la información sobre profesiones almacenadas en el ordenador. Una vez cotejadas las características del sujeto con la información disponible, el ordenador imprime todas las ocupaciones que se adaptan a las mismas. La interacción entre sujeto y ordenador es inexistente.

La segunda generación resulta algo más fructífera al dedicarse al almacenamiento de la información para crear las primeras grandes bases de datos. Ya es posible la interacción directa entre sujeto y ordenador. Como ejemplos más representativos destacan en el contexto americano los programas CVIS, CIS y GIS o en Europa el programa francés SOCRATE.

La tercera generación se caracteriza por la irrupción de los sistemas de orientación vocacional, denominados CACGS en inglés (Computer Assisted Career Guidance Systems). A nivel teórico se basan en el enfoque de desarrollo vocacional de forma que entienden la orientación como un proceso, motivo por el cual incorporan -además de un gran bloque informativo- pruebas de evaluación, interactivas, simulaciones y módulos de instrucción. El desarrollo más conocido es DISCOVER.

Con la cuarta generación se inicia un proceso de adaptación de los programas creados para los grandes ordenadores centrales (como los conocidos SIGI y DISCOVER) a los ordenadores personales. A pesar de que no se producen grandes cambios, sí existe un vuelco hacia una población más diversa y se posibilita que las aplicaciones que antes sólo funcionaban en grandes equipos ahora se puedan utilizar en los centros educativos o en las casas. Es preciso destacar esta característica que marca el desarrollo posterior de la orientación al permitir su generalización a toda la sociedad.

Existe una quinta generación, en la que se están desarrollando las capacidades cada día mayores de los equipos informáticos, que están permitiendo la aparición de programas muy potentes. Se trata de sistemas multimedia interactivos basados en el uso de gráficos, audio y vídeo, que facilitan representaciones mentales sobre elecciones vocacionales.

Por nuestra parte nos atrevemos a esbozar una sexta generación que viene marcada por la integración de los distintos recursos tecnológicos en entornos multimedia en red, la extensión a todos los sectores sociales de Internet y la ampliación de sus capacidades de transmisión de datos a través de las denominadas tecnologías de acceso de banda ancha. Las líneas RDSI y ADSL construidas sobre las tradicionales de hilo de cobre junto con las nuevas de cable de fibra óptica, ofrecen altas capacidades en la transmisión y recepción de datos. En concreto, estas últimas permiten la integración de teléfono, televisión e Internet, uno de los grandes alicientes de la nueva generación. Esta revolución de la información y del conocimiento facilita la orientación tutorizada en las mismas casas, el fácil acceso a las fuentes documentales, la búsqueda de empleo y un sinfín de posibilidades, algunas de ellas todavía por explotar. Por ejemplo, las simulaciones inteligentes en las que el usuario se recrea desempeñando un determinado trabajo o la tutorización telemática interactiva en tiempo real mediante videoconferencia. Este último instrumento 
se percibe ya como una necesidad en un futuro próximo, dado que sus características técnicas permiten visualizar e intervenir en contextos que de otra forma serían inalcanzables para el orientador (Cogoi, 2002).

Todo lo expuesto nos lleva a una importante proyección de futuro de las NTIC en la orientación (Walz, 1991):

«El uso de la tecnología y los ordenadores constituyen prometedores instrumentos que contribuyen a la mejora de los procesos y productos de la orientación. Están llamados a tener un impacto considerable en el futuro».

Esta idea, compartida por Sanz (2001: 199) cuando cita entre las tendencias más prometedoras la «mayor utilización de los medios tecnológico-matemáticos», la asumimos también como nuestra con la precisión añadida de que no podemos estar hablando de futuro toda la vida -algunas citas tienen ya más de una década- y comprobar día a día cómo la posición de los profesionales de la orientación ante los ciudadanos del mañana pierde terreno en relación con el resto de las disciplinas en la adopción de recursos tecnológicos que favorezcan la calidad de la intervención orientadora.

A un nivel más teórico, encontramos como precedente del MT la inclusión de los recursos tecnológicos por Morril y otros (1974) en su cubo tridimensional, cuando las tecnologías eran más un proyecto que una realidad. Ya intuían estos autores su influencia en la orientación y, por tal motivo, las toman en consideración dentro de la dimensión «Métodos de la intervención».

En cuanto a la opinión de expertos en las jornadas, conferencias o congresos sobre orientación llevados a cabo hasta la fecha, existen claras referencias a la utilización de los ordenadores, convirtiéndose en los últimos años en un tema recurrente tanto en ponencias como en comunicaciones. Según Repetto y otros (1994: 863) en 1979 se inician las primeras demostraciones de programas informáticos aplicados a la orientación en un congreso internacional celebrado en Alemania y, unos años más tarde, en 1984 «la Asociación Internacional de Orientación Escolar y Profesional (AIOSP) reconoció públicamente la importancia del desarrollo de la orientación vocacional asistida por ordenador».

En la Segunda y Tercera Conferencia Europea de Servicios de Orientación (Computers in Careers Guidance) celebradas respectivamente los años 1989 y 1992, como claves en el desarrollo de las aplicaciones informáticas a la orientación (Rodríguez Espinar y otros, 1993; Álvarez González, 1995), se marcaron los siguientes objetivos:

1. Adaptarse a las nuevas tecnologías.

2. Apoyar los intercambios tecnológicos entre países de la Comunidad.

3. Diseñar lo que podría ser una acción común en este campo.

En ambas Conferencias se abogó por la necesidad de potenciar la utilidad de los ordenadores en la orientación, priorizando la potenciación de proyectos internacionales para estimular la realización de programas informáticos. Ya se advirtió en ellas que la figura del orientador debería estar presente en estos programas desempeñando la función de consultor, aportando su opinión especializada a nivel de diseño, así como resolviendo los problemas específicos y ayudando al orientando en la reflexión.

Para no abundar en exceso en las llamadas a la potenciación del uso de las NTIC en la orientación que a partir de estos dos congresos anteriores se han venido produciendo con 
periodicidad -por ejemplo, el Congreso Internacional de la AIOSP de 1991 o la $4^{\text {a }}$ Conferencia Europea sobre Orientación y TIC de 1997- que han pretendido ir calando en la opinión de expertos y profesionales, citamos a continuación las principales líneas de trabajo desarrolladas en la « $5^{\mathrm{a}}$ Conferencia Europea sobre Tecnologías de la Información y la Comunicación en Orientación» celebrada en junio de 2001, abordando los siguientes núcleos temáticos: herramientas y test interactivos en la web, la relación cliente-orientador, el desarrollo y utilidad de los recursos de información, el acceso a las tecnologías de la información y la orientación por la red, y el nuevo papel de los orientadores y su función en la formación inicial y posterior. Estas cuestiones se trataron a través de cuatro grandes líneas de trabajo: el aprendizaje a lo largo de toda la vida, el futuro desarrollo de la orientación por red, la perspectiva del cliente y las redes profesionales como herramienta común. Esto prueba la gran expectación que se está produciendo en torno a las posibilidades que ofrece Internet y sus consecuencias en la orientación del futuro. Por este motivo, la conferencia elaboró una serie de propuestas concretas relacionadas con la orientación por la red con la finalidad de hacerlas llegar a los responsables políticos de la Comisión Europea.

Finalmente, hacemos un balance de los temas a los que más atención se está prestando en los últimos años (Marín y Rodríguez Espinar, 2001):

- Desarrollo de la carrera e Internet (Harris-Bowlsbey y otros, 1998; Offers y Watts, 1997), así como los efectos en el desarrollo de la identidad vocacional y conductas exploratorias (Mau,1999).

- Uso de Internet en el diagnóstico de la carrera (Oliver y Zack,1999).

- Guías informativas para el uso de Internet en el ámbito de la orientación (Hartman, 1996; Wolfinger, 1998).

- Guías-sistemas de información, en especial información profesional y ocupacional (Peterson y otros, 1999).

- Cuestiones éticas en el uso de Internet (NCDA, 1996, 1997; NBCC, 1997).

- Librerías virtuales (Bleuer y Walz, 1998).

- Problemas asociados al diseño y uso de TIC (Bartram,1997; Krumboltz y Winzelberg, 1997; Sampson, 1999; Sampson y otros, 1997).

Como vemos, los orígenes del MT se inscriben en la toma de conciencia de las posibilidades que ofrecen las NTIC en la orientación, de manera especial los desarrollos informáticos, que son los que hasta ahora han tenido una mayor incidencia en la misma. Las referencias a lo largo de este análisis histórico al MT son prácticamente nulas y han quedado supeditadas a citas puntuales basadas en el uso de los ordenadores en la orientación y exentas todas ellas de un desarrollo sistemático (Rodríguez Espinar y otros, 1993; Álvarez Rojo, 1994; Álvarez González, 1995; Bisquerra y Álvarez González, 1996; Martínez González, 1998; Lázaro y Mudarra, 2000; Sobrado y Ocampo, 2000).

\section{Principales notas distintivas}

La anterior revisión histórica apenas si nos ha aportado datos referidos a la formación del MT. Es más, la literatura especializada sólo presenta un pequeño avance en el mismo al 
citar como única característica la utilización de recursos tecnológicos específicos (Rodríguez Espinar y otros, 1993; Álvarez González, 1995; Bisquerra y Álvarez González, 1996; Martínez González, 1998; Sobrado y Ocampo, 2000). Su reciente inclusión entre la terminológica técnica de orientación, como advertíamos al comienzo de este apartado, hace que algunos autores piensen que todavía no existe como tal.

Admitiendo como punto de partida que el MT sólo se activa si funciona a través de uno o más modelos - de no ser así no estaríamos hablando de modelo sino de una simple aplicación de técnicas o recursos más o menos potentes a la orientación- es preciso sobrepasar el actual estancamiento conceptual y definir con claridad sus principales rasgos característicos.

La teoría de la comunicación, las teorías del aprendizaje y la teoría general de sistemas son las tres ciencias sociales que han venido apoyando las propuestas tecnológicas aplicadas a la educación (De Pablos, 1996), que son el referente más inmediato antes de su llegada a la orientación. Dentro de la teoría de la comunicación se han realizado numerosos estudios sobre los medios de comunicación de masas (prensa, radio, televisión) basados en los efectos de esos medios sobre la población.

Con respecto a la elaboración de programas informáticos, Gros (1997) ha señalado que las teorías del aprendizaje tanto de corte conductista, cognitivista o constructivista están en la base de toda elaboración de software que se realiza con una intencionalidad educativa, ya sea utilizando modelos sistemáticos, no lineales o hipertextuales. Así, existen ya diferentes programas tutoriales, simulación de la toma de decisiones vocacionales, de ejercitación, hipertextos e hipermedias que están revolucionando en cierto modo el proceso de intervención tecnológica en el campo de la orientación.

Finalmente, desde la teoría general de sistemas se toman como referencia numerosos modelos que están en la base de diversas aplicaciones tecnológicas. Sus aportaciones constituyen el punto de partida para la elaboración de gran cantidad de ingenios tecnológicos aplicados a la educación, puesto que el comportamiento de éstos tiene mucho más que ver con las estructura de retroalimentación que los caracteriza que con la entidad de sus componentes físicos. Pero es más, los propios productos tecnológicos (vídeos, programas informáticos, etc.) no son sino sistemas reales que sometidos a estudio pueden a su vez aportar modelos sistémicos susceptibles de enriquecer el marco teórico donde se insertan.

Esta reflexión conceptual nos aporta la perspectiva de que los recursos tecnológicos tienen un diseño basado en una determinada posición psicopedagógica, por lo que habrán de ser analizados y estudiados previamente por el orientador y por el equipo que llevará a cabo la intervención orientadora.

Las características de los recursos tecnológicos son determinantes de algunas fases del MT, pero están supeditados siempre al desarrollo de éste, que a su vez complementa la labor desarrollada por el orientador, que hará revertir en él procedimientos, estrategias y sistemas más dinámicos, participativos y reflexivos en el tratamiento de la información (Álvarez González, 1995).

Álvarez Rojo (1994: 221) realiza una escueta definición del MT al afirmar que «el modelo tecnológico se basa en la secuenciación Necesidades-Objetivos-Recursos-Estrategias y es ampliamente conocido...» 
A este respecto, Bisquerra (1996: 156) ha señalado algunas características del MT que lo diferencian del resto de modelos:

"El modelo tecnológico consiste en la utilización de los "mass media" (prensa, radio, televisión, vídeo, programas informáticos, etc.). Fijémonos en que el modelo clínico atiende a los clientes uno a uno; el modelo de servicios puede atender a grupos, igual que el modelo de programas; con el modelo de consulta un mismo orientador puede atender de forma indirecta a conjuntos de grupos, cada uno de los cuales es atendido por el mediador. Con el modelo tecnológico se puede atender simultáneamente a cantidades ingentes de personas».

De forma más concreta podemos decir que el MT se basa en la integración de NTIC en un programa comprensivo de intervención dirigido preferentemente a la prevención y al desarrollo, que despliega sus funciones alternando las dimensiones dentro-fuera del contexto donde se lleve a cabo, directa-indirecta e individual-grupal. Según los casos, puede adoptar determinadas características de otros modelos de intervención.

El hecho de que las raíces del MT se sitúen en otros modelos no le quita identidad propia, sino que fortalece su capacidad de adaptación a la naturaleza de cada intervención. Desde nuestro punto de vista, será a través de programas comprensivos integrados en el currículum como mejores resultados consiga.

Ni la figura ni las funciones del orientador quedan debilitadas en el MT, sino que sufren una reconversión:

- Realiza preferentemente tareas de consultor, al estar presente en el uso de las nuevas tecnologías aplicadas a la orientación aclarando dudas, resolviendo problemas, comentando la información y ayudando a los usuarios a la toma de decisiones.

- Es un mediador material de los aprendizajes y del desarrollo de los destinatarios de la orientación.

- La intervención adopta un carácter transversal, preventivo y de desarrollo.

- Coordina las labores que desarrollarán los distintos agentes de intervención que complementarán su labor.

- Estudia los diferentes desarrollos existentes en NTIC y analiza sus efectos potenciales en la orientación.

Una mención aparte merece su papel de técnico o especialista conocedor y dominador de las NTIC, de forma más concreta de aquellos desarrollos diseñados o adaptados a la orientación. Sanz y Sobrado (1998: 48), ya se refirieron a este rol tras definir al orientador como un agente de cambio socioeducativo:

«Emplear nuevos recursos multimedia y tecnologías innovadoras en el desarrollo de sus tareas como son el vídeo, la informática, internet, redes de información, etc.».

Es importante también destacar el apoyo que tiene el orientador en este modelo en otras figuras, como profesores y tutores (incluso, padres), que en determinados momentos asumen funciones propias.

Con estas peculiaridades, el MT provoca un tipo de orientación que exige el apoyo de un medio tecnológico que actúa de intermediario entre el agente de la intervención (profesional, empresa, institución, etc.) y la persona objeto de la acción orientadora. El orientador es el que diseña y/o asesora en la relación que se produce, lo que, como hemos dicho, no 
significa que su papel sea secundario o marginal. Pero serán las aportaciones tecnológicas las que influirán decididamente en las características del medio elegido para la intervención y éste en el proceso orientador.

El MT puede adoptar distintos formatos, con la característica común de ser sistemas interactivos capaces de proporcionar a los estudiantes estímulos y respuestas que serán completados de forma simultánea por simulaciones de todo el proceso, de tal forma que no sólo ofrezca una representación de la realidad, sino que permita servir de ejemplo a seguir al alumno. El orientador aparece cuando el proceso lo demanda o lo requiere el propio alumno. En el MT los procesos de tipo administrativo o burocrático, como pueden ser la administración y corrección de pruebas, sugerencias aventuradas, argumentaciones rápidas, etc. quedan en un segundo plano, puesto que cuando el alumno llega hasta él, ya conoce el problema y ha dispuesto de tiempo suficiente para preparar sus posibles soluciones (Pantoja y Campoy, 2001).

El medio más característico del MT es el informático y el telemático, ambos funcionan mayoritariamente sobre la plataforma PC. Maze (1984) ha señalado algunos componentes que habrán de tener los ordenadores aplicados a la orientación:

- Componentes didácticos: Podrían tratarse el proceso de planificación profesional, la decisión de elaboración, la estructura del mundo del trabajo, obtención de objetivos financieros, escribiendo un resumen y entrevistando.

- Componentes de valoración propia: Aquí entrarían las utilidades que permiten administrar y marcar instrumentos de evaluación. Ejercicios de cientos de items pueden ser administrados por los ordenadores, marcados y resueltos de forma instantánea.

- Selección profesional: Los ordenadores ofrecen grandes facilidades para clasificar profesiones y organizarlas en categorías.

- Componentes de conocimientos: Complementan a los anteriores al incluir información acerca de profesiones, programas educativos y materiales bibliográficos.

Las ventajas que aporta el MT, tanto si se basa en medios informáticos como si lo hace en audiovisuales, son las siguientes:

- Favorece la motivación de los estudiantes al encontrar más atractiva la acción orientadora.

- Mejora la comprensión de conceptos.

- Amplía los horizontes del contexto donde se enmarca la orientación al permitir que ésta llegue a cualquier punto del exterior: conocimiento de profesiones, empresas, estudios universitarios, empleo, etc.

- Facilita el trabajo al presentar la información de una forma no lineal y permitir saltar de unos contenidos a otros en función de las necesidades personales.

- Permite una adaptación bastante precisa a los intereses personales y al ritmo de trabajo de cada alumno.

- Se adapta a la orientación individual al favorecer en los alumnos respuestas individualizadas a sus demandas.

- Favorece las intervenciones grupales:

- Mejora el contexto social. 
- Amplía la esfera de relaciones.

- Facilita el sentimiento de grupo cohesionado.

- Integra los comentarios y opiniones de los compañeros.

- Permite la transmisión de valores.

- El proceso orientador se enriquece al facilitar los recursos tecnológicos que los estudiantes aprendan de sus errores en los procesos de simulación y modelado que se producen.

A modo de resumen, las principales notas definitorias del MT son:

- Sitúa la orientación en un escenario más amplio que el contexto donde se ubica, proyectándose en toda su extensión a todos los sectores: alumnos, familias, profesionales de la orientación y tutores.

- Facilita el intercambio de toda clase de información entre los implicados en el acto orientador.

- Confiere autonomía a los implicados.

- Permite un gran número de posibilidades de desarrollo de la acción orientadora.

- Se puede integrar en otros modelos y en el currículum de orientación.

\section{Cambios en el rol del orientador y fases en el desarrollo del MT}

La principal implicación práctica que tiene el MT es el cambio que provoca en el rol del orientador, característica básica del mismo. Los roles más significativos son: consultor, mediador, asesor, coordinador. Sanz (2001: 200-201) ha señalado al respecto:

«El rol del orientador, pues, debería ser conectar las necesidades con los recursos disponibles. Por ejemplo, la posibilidad de utilizar programas de "peer facilitators" en un centro educativo constituye un magnífico recursos para "ampliar" la acción profesional de la orientación a un mayor número de usuarios. En el futuro, estos programas, así como la utilización de medios tecnológicos aplicados al funcionamiento de la orientación y el implicamiento directo de padres y voluntarios de la comunidad, constituirán intervenciones que tendrán que tener su lugar dentro de la escuela».

Sobrado (2001) también ha expuesto que las NTIC tienen que llegar a la orientación en forma de nuevos roles a desarrollar como demanda social:

"Las reformas en la educación y en el trabajo, los cambios sociales, económicos y tecnológicos con un ritmo muy acelerado en la realidad actual, plantean la exigencia de la innovación en los roles, perfiles y funciones de la orientación profesional en sus conexiones con los contextos sociales, educativos y laborales».

Asumido este cambio de rol, las implicaciones prácticas del MT precisan de una concreción detallada de las tecnologías y de los desarrollos de las mismas que se utilizarán. Aquí no vale la improvisación, por lo que es de suma importancia que se valoren previamente, entre otros, los pros y contras que conlleva la utilización del material elegido y las necesidades que precisa para un correcto funcionamiento. 
Para evitar que el MT quede simplemente en la utilización de NTIC, proponemos a continuación una posible metodología de trabajo estructurada y sistematizada en varias fases (gráfico 3):

1. Inicio de la acción orientadora por iniciativa del orientador, algún miembro de la comunidad educativa (padre, profesor o alumno) o por mandato institucional.

2. Estudio de la naturaleza de las acciones a emprender. No es igual idear el plan para atajar un problema o como propuesta de prevención o desarrollo de aspectos compartidos por el grupo.

3. Elección del modelo o modelos de orientación adecuados. Aunque nuestra postura es actuar, siempre que sea posible, mediante programas comprensivos, no hay que descartar cualquier otra posibilidad derivada de la naturaleza de la propia intervención.

4. Análisis de los recursos tecnológicos disponibles que traten el tema en cuestión para definir en función de los mismos:

- Revisar posibles evaluaciones previas de los mismos.

- Roles y funciones de personas implicadas.

- Momentos de utilización: cronograma.

- Dimensiones sociales de la intervención: individual y grupal (compañeros, profesor u orientador, padres, etc.).

- Lugar o lugares en los que se desarrollarán (colegio, casa, etc.).

- Utilidad de todos los apartados de que consta el recurso o sólo de algunas partes del mismo.

- Posibilidades de adaptación.

- Otros.

5. Elección de los recursos (tecnológicos y de lápiz y papel).

6. Diseño del programa comprensivo. Dada la flexibilidad de las NTIC y su escasa durabilidad -salen versiones nuevas de forma continua- hay que dejar una puerta abierta, tanto en el diseño como en la implementación, a un imprescindible proceso de feedback que permita incorporar las posibles novedades del mercado y adaptar las mismas, en su caso, a las necesidades que se planteen. Estará previsto si la secuencia que siguen los recursos tecnológicos elegidos se llevará a cabo completamente o, por el contrario, se efectuarán saltos en función de los objetivos establecidos. Bien entendido que se tratará muy probablemente de recursos de tipo estandarizado, es decir, no diseñados específicamente para atender ese plan de intervención. Los recursos abiertos, como es el caso de Internet, tendrán definida con claridad su metodología de uso (objetivos, contenidos a tratar, temporalización, tiempos de uso, lugar, forma de agrupamiento, etc.).

7. Implementación. Existirá una previsión de cómo se llevará a cabo la intervención, por quién o quiénes, cuándo se desarrollará y dónde. En cada una de estas variables se asignará un agente de intervención concreto.

8. Evaluación y propuestas de depuración. La evaluación se efectuará de forma que englobe a todos los participantes. La calidad de la intervención dependerá de que este proceso sea riguroso y técnico a la vez. Las propuestas de depuración se diri- 
girán a la empresa responsable de diseñar el programa o al propio plan de intervención si incorpora recursos tecnológicos abiertos, tales como Internet, vídeo o televisión. De la rigurosidad de esta fase depende que la próxima vez que se ponga en marcha el MT a través de un plan estructurado hayan desaparecido los problemas detectados.

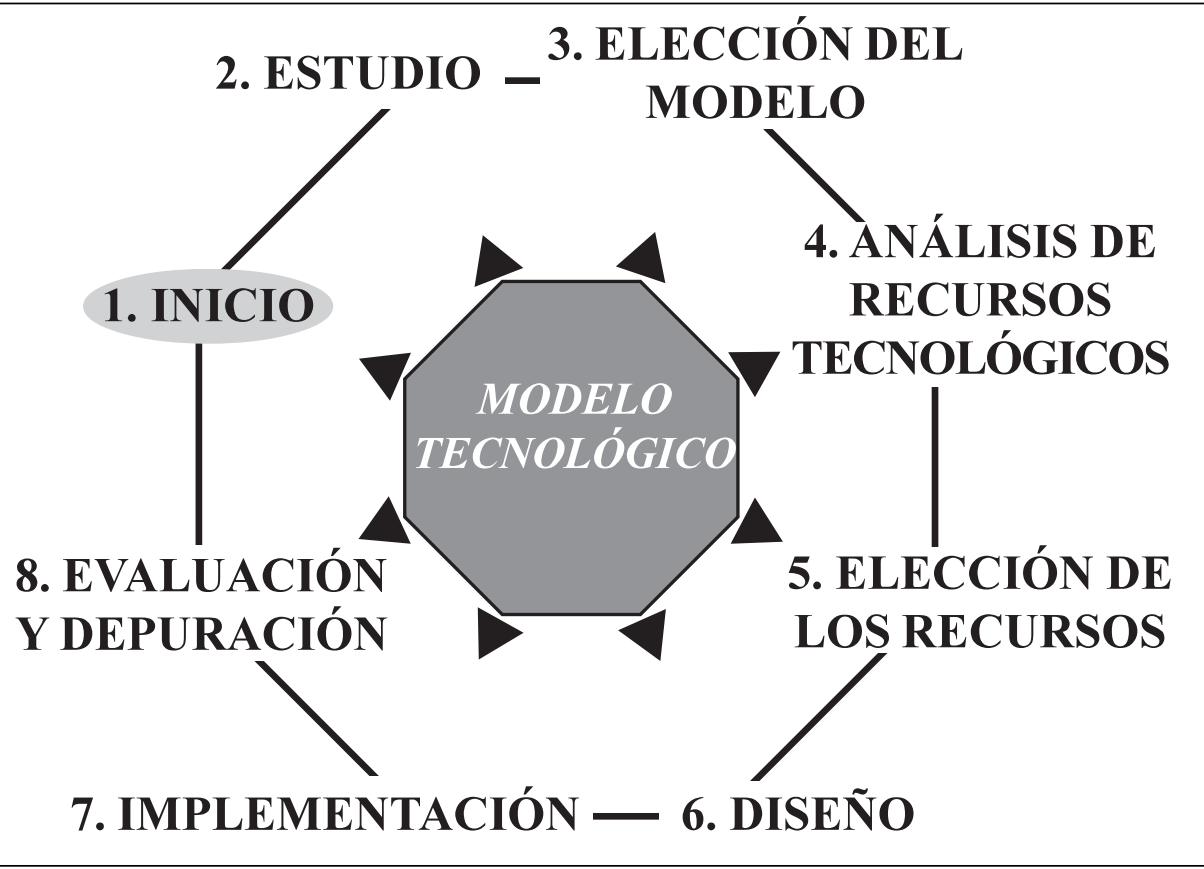

GRÁFICO 3.

Fases en el Modelo Tecnológico.

Antes de inciar el trabajo con las NTIC es necesario realizar algunas previsiones:

- Decidir qué aplicación concreta se va a utilizar.

- Preparar la aplicación y el material asociado a la misma.

- Analizar las formas de utilización.

- Reservar el lugar de uso dentro del centro.

\section{Principales limitaciones del MT}

El principal inconveniente que encuentra el MT es el mismo que tiene la informática educativa. Se trata de costosos sistemas informáticos que siembran dudas en las empresas encargadas de desarrollarlos, por el temor que suscita una probable baja demanda. Por esta razón algunas de las aplicaciones existentes están obsoletas, se basan todavía en el viejo 
MS-DOS, apenas si ofrecen interactividad y no se actualizan. En estos momentos se puede afirmar que la intervención orientadora a través de los medios tecnológicos está limitada precisamente por el escaso nivel de los programas existentes (Pantoja y Campoy, 2001).

Otro obstáculo añadido, ya comentado con anterioridad, es el hecho de que se asocie MT a la simple utilización de recursos tecnológicos, también presente en la educación. Esto suele llevar al desencanto a quienes piensan que con entregar un disco a sus alumnos con un programa sobre orientación vocacional, por ejemplo, ya se activa un proceso de mejora de la calidad de la orientación y, por consiguiente, de sus resultados finales.

Un problema que afecta a la práctica es la estandarización de las aplicaciones tecnológicas, preocupadas por abordar una amplio abanico de posibilidades, lo que repercute en una escasa adaptación a los contextos y situaciones. Otro motivo más para realizar un análisis riguroso de las mismas antes de su utilización.

El modelo didáctico subyacente en los programas generados con las NTIC supone un handicap de gran calado en los resultados finales. Los autores de cada aplicación conciben y desarrollan las mismas en función de una determinada concepción psicopedagógica. Así, encontramos propuestas basadas en el modelo conductista-tecnológico en el que priman los mecanismos de estímulo-respuesta; otras que, desde una perspectiva genético-cognitiva, se apoyan en la presentación de experiencias, buscando estimular la curiosidad y el descubrimiento; o las que, desde una posición socio-histórica, convierten el mundo exterior en el modelo de la actividad interna. Estos distintos posicionamientos conceptuales pueden condicionar un correcto desarrollo del MT si el orientador no hace una valoración previa de los posibles condicionantes. Lo ideal sería que las aplicaciones tecnológicas tuviesen la suficiente flexibilidad y adaptabilidad para permitir que los estudiantes pudiesen moverse por las mismas con libertad y autonomía en un proceso de interacción personalizada. Esto no es así en la mayoría de los casos.

En otro ámbito también destaca la falta de una conciencia de la política educativa española y de las autoridades ante la necesidad de aplicar programas de orientación para la carrera en todos los niveles educativos, insertados dentro del currículum (Repetto, 2001). Esta perspectiva de orientación a lo largo del ciclo vital podrá llevar a los orientadores profesionales a acceder a herramientas que le facilitasen el proceso de orientación y la autoorientación de los alumnos. Este último aspecto es una característica esencial en el MT.

\section{Referencias bibliográficas}

Álvarez González, M. (1995). Orientación profesional. Barcelona: Cedecs.

Álvarez González, M. y Rodríguez Espinar, S. (2000). Cambios socio-educativos y orientación en el siglo XXI: Nuevas estructuras, roles y funciones. Actas del XII Congreso Nacional y I Iberoamericano de Pedagogía. Tomo I. Ponencias. Madrid: SEP, pp. 637-686.

Álvarez González, M. y Rodríguez Espinar, S. (2001). Nuevas estructuras, roles y funciones de la orientación. En A. Pantoja, T. J. Campoy y A. Cañas (Coords.), Nuevas perspectivas de la orientación educativa. Jaén: Cámara Oficial de Comercio e Industria de la provincia de Jaén, pp. 43-83.

Álvarez Rojo, V. (1994). Orientación educativa y acción orientadora. Relaciones entre la teoría y la práctica. Madrid: EOS. 
Benavent, J. A. y Fossati, R. (1998). El futuro de los modelos de la Orientación Psicopedagógica. En R. Bisquerra (Coord.), Modelos de orientación e intervención psicopedagógica. Barcelona: Praxis, pp. 457-467.

Benavent, J. A. (1999). La orientación psicopedagógica en el umbral del S. XXI: Una mirada al futuro. Revista Española de Orientación y Psicopedagogía, 10 (17), 53-62.

Bisquerra, R. y Álvarez González, M. (1996). Modelos de intervención en orientación. En M. Álvarez González y R. Bisquerra (Coords.), Manual de orientación y tutoría. Barcelona: Praxis, pp. 331-351.

Bisquerra, R. (1996). Orígenes y desarrollo de la orientación psicopedagógica. Madrid: Narcea.

Bleuer, J.C. y Walz, G.R. (1998) ERIC/CASS virtual libraries: On-line resources for parents, teachers and counselors. En J.M. Allen (ed.), School counseling: New perspectives \& practices. Greensboro, N.C.:ERIC/CASS, University of North Carolina at Greensboro, pp. 77-185.

Castells, M. (1997, 1998). La era de la información: Economía, sociedad y cultura. Vol. I: La sociedad red. Vol. 2: El poder de la identidad. Vol. 3: Fin del milenio. Madrid: Alianza.

Castells, M. (2001). Materiales para una teoría preliminar sobre las sociedades de redes. Revista de Educación, número extraordinario, 41-58.

Cogoi, C. (2002). Videoconferenza e orientamento. Ambiti di applicazione ed esempi di buone practiche. Revista Española de Orientación y Psicopedagogía, 13 (1), 5-15.

Daniel, R.W y Weikel, W.J. (1983). Trends in counseling: A Delphi Study. Personnel and Guidance Journal, 61, 327-331.

Delors, J. y otros (1996). La educación encierra un tesoro. Madrid: Santillana

Echeverría Ezponda, J. (1999). Los señores del aire: Telépolis y el tercer entorno. Barcelona: Destino.

Echeverría Ezponda, J.(2001b). Educación y Sociedad de la Información. Revista de Investigación Educativa, 19 (2), 277-289.

Fernández, L.A. (1998). En.red.ando. Barcelona: Zeta Ediciones.

Gros, B. (1997). Diseños y Programas Educativos. Barcelona: Ariel.

Harris-Bowlsbey, J. (1995). Career planning and computer-based systems: Models of integration. Guidance \& Counselling, 10 (4), 25-30.

Harris-Bowlsbey, J. y otros (1998). The Internet: A tool for career planning. Columbus, OH: National Carer Development Association.

Hartman, K. (1996). Internet Guide for College-Bound Students. New York: College Entrance Examination Board.

Herr, E.L. y Cramer, S.H. (1992). Career guidance and counseling through the life span. Nueva York: Harper Collins.

Krumboltz, J.D., y Winzelberg, A. (1997). Technology applied to learning and group support or career-related concerns. Career Planning and Adult Development Journal, 13, 101-110.

Lázaro, A. y Mudarra, M.J. (2000). Análisis de los estilos de orientación en equipos psicopedagógicos. Contextos educativos, 3, 253-280.

Levy, P. (1999). ¿Qué es lo virtual? Barcelona: Paidós.

Marcelo, C. (2000). El profesor de enseñanza primaria. Actas del XII Congreso Nacional y I Iberoameriacano de Pedagogía: Hacia el tercer milenio: Cambio educativo y educación para el cambio. Madrid: SEP, pp. 392-420

Marco, R. (1995). La tecnología informática en el asesoramiento vocacional. En F. Rivas, Manual de asesoramiento y orientación vocacional. Madrid: Síntesis, pp. 383-419. 
Marín, M.A. y Rodríguez Espinar, S. (2001). Prospectiva del diagnóstico y la orientación. Revista de Investigación Educativa, 19 (2), 315-362.

Martínez González, M. de Codés (1998). Orientación Escolar. Madrid: Sanz y Torres.

Mau, W. C. (1999). Effects of computer-assisted career decision making on vocational identity and career exploratory behaviors. Journal of Career Development, 25, 261-274.

Maze, M. (1984). How to Select a Computerized Guidance System. Journal of counseling and development, 63 (3), 158-161.

Morrill, W.H., Oetting, E. R. y Hurst, J. C. (1974). Dimensions of counselor functioning. Personnel and guidance journal, 52, 354-359.

National Board for Certified Counselors and the Council for Credentialing and Education (1997). Standards for the ethical practice of WEB Counseling. Greensboro, NC: Author.

National Career Development Association (1996). An ethics statement on the use of Internet in providing career services. Columbus, $\mathrm{OH}$ : Author.

National Career Development Association (1997). NCDA guidelines for the use of theInternet for provision of career information and planning services. Alexandria, VA: Author.

Negroponte, N. (1995). El mundo digital. Barcelona: Ediciones B.

Offer, M. y Watts, A. G. (1997). The Internet and career work. NICEC Briefing. Cambridge; UK: National Institute for Careers Education and Counseling, Sheraton House, Castle Park, Cambridge CB3 OAX.

Oliver, L. W. y Zack, J. S. (1999). Career assessment on the Internet: An exploratory study. Journal of Career Assessment, 7, 323-356.

Pantoja, A. (2001). Orientación educativa, nuevas tecnologías y sociedad de la información. Actas del Congreso Internacional Virtual de Psicología Educativa (CIVPE). Islas Baleares: Cibereduca. Edición en CD-Rom. ISBN: 84-7632-713-7.

Pantoja, A. y Campoy, T. J. (2001). Un modelo tecnológico de orientación universitaria. En L. M. Villar Angulo (Coord.), La universidad. Evaluación educativa e innovación curricular. Sevilla: ICE de la Universidad de Sevilla, pp. 95-128.

Peterson, N. G. y otros (eds.) (1999). An occupational information system for the 21th century: The development of $O^{*}$ Net. Washington, DC: American Psychological Association.

Rayman, J. R. (1990). Computers and career counseling. En W. B. Walsh y S. H. Osipow (Eds.), Career counseling. Contemporany topics in vocational counseling. Hillsdale, NJ: Erlbaum.

Repetto, E. (2001). El papel de los programas de orientación profesional: claves facilitadoras de la orientación. Número monográfico 20 de Educaweb sobre La orientación académica y profesional. Dirección electrónica en Internet: http:/www.educaweb.com/esp/servicios/monografico/orienta/opinion12.asp

Repetto, E. y Malik, B. (1998). Nuevas tecnologías aplicadas a la orientación. En R. Bisquerra (Coord.), Modelos de orientación e intervención psicopedagógica. Barcelona: Praxis, pp. 363-374.

Repetto, E. y otros (1994). Orientación educativa e intervención psicopedagógica. Madrid: UNED.

Rodríguez Espinar, S. (coord.) y otros (1993). Teoría y práctica de la orientación educativa. Barcelona: PPU.

Rodríguez Roselló, L. (2001). Tecnologías de la sociedad de la información para educación y la formación: Hacia una red europea del conocimiento. Conferencias inaugural del congreso internacional, La educación en internet e internet en la educación. Madrid, 14-16 de diciembre.

Sampson, J. C. y Reardon, R. C. (1990). Enhancing the design and use of computer-assisted career systems. Washington, DC: National Career Development Association. 
Sampson, J. C. y Reardon, R. C. (1991). Current development in computer-assisted career systems. British Journal of Guidance and Counselling, 19 (2), 113-128.

Sampson, J. P., Jr. (1999). Integrating Internet-Based Distance Guidance With Services Provided in Career Center. The Career Development Quarterly, 47 (3), 243-254.

Sampson, J. P. y otros (1997). Counseling on the informatión highway: Future possibilities and potential problems. Journal of Counseling and Development, 75, 203-212.

Sancho, J. M. (2000). Las nuevas tecnologías como campo de investigación: de la simplicidad a la complejidad. Actas del XII congreso Nacional y I Iberoamericano de Pedagogía: Hacia el tercer milenio: Cambio educativo y educación para el cambio. Tomo I. Ponencias. Madrid: SEP, pp. 301-321.

Sanz, R. (2001). Orientación psicopedagógica y calidad educativa. Madrid: Pirámide.

Sanz, R. y Sobrado, L. (1998). Roles y funciones de los orientadores. Revista de Investigación Educativa, 16 (2), 25-57.

Sobrado, L. (2001). Los roles que la sociedad actual exige a los orientadores son los de ser agentes de cambio social, formativo y profesional. Número monográfico 26 de Educaweb dedicado a La orientación académica y profesional. Dirección electrónica en Internet: http://www.educaweb.com/esp/servicios/monografico/orientacion/opinion2.asp

Sobrado, L. y Ocampo, C. (2000). Evaluación psicopedagógica y orientación educativa. Barcelona: Estel.

Solé, I. (1998). Orientación e intervención psicopedagógica. Barcelona: ICE/Horsori.

Telefónica (2001). La Sociedad de la Información en España. Perspectiva 2001-2005. Dirección electrónica en Internet: http://www.telefonica.es/sociedaddelainformacion.

Terceiro, J. B. (1996).Socied@ddigit@l.Del homo sapiens al homodigitalis. Madrid: Alianza Editorial.

Vélaz de Medrano, C. (1998) Orientación e intervención psicopedagógica. Concepto, modelos, programas y evaluación. Archidona: Aljibe.

Walz, G. (1991). Future focused generalizations on counseling. En G. Walz y otros, Counseling Futures. Ann Arbor, MI: ERIC Counseling and Personnel Services Clearinghouse.

Wolfinger, A. (1998). The quick Internet guide to career and college information. Indianapolis, IN: JIST works, Inc.

Fecha de recepción: 2 -IX-02

Fecha de revisión: 11-X-02

Fecha de aceptación: 5-XI-02 